\title{
Noise Expresses Exponential Growth under Regime Switching
}

\author{
Guangda $\mathrm{Hu}^{1}$, Mingzhu Liu ${ }^{2}$, Xuerong $\mathrm{Mao}^{3, *}$, Minghui Song ${ }^{2}$ \\ ${ }^{1}$ Department of Automation, \\ School of Information Engineering, \\ University of Science and Technology Beijing, \\ Beijing 100083, China \\ 2 Department of Mathematics, Harbin Institute of Technology, \\ Harbin, China. \\ ${ }^{3}$ Department of Statistics and Modelling Science, \\ University of Strathclyde, Glasgow G1 1XH, U.K.
}

\begin{abstract}
Consider a given system under regime switching whose solution grows at most polynomially, and suppose that the system is subject to environmental noise in some regimes. Can the regime switching and the environmental noise work together to make the system change significantly? The answer is yes. In this paper, we will show that the regime switching and the environmental noise will make the original system whose solution grows at most polynomially become a new system whose solution will grow exponentially. In other words, we reveal that the regime switching and the environmental noise will exppress the exponential growth.
\end{abstract}

Key words: Brownian motion, Markov chain, stochastic differential equation, stochastic perturbation, exponential growth, polynomial growth.

AMS Classifications: $60 \mathrm{H} 10,93 \mathrm{E} 15$

\section{Introduction}

One of the important problems in the study of stochastic differential equations is to reveal whether stochastic perturbations have significant effects on the underlying systems which are described by ordinary differential equations. In this paper we will consider the following problem: Can the regime switching and the environmental noise work together to make a given system whose solution grows at most polynomially become a new system whose solution will grow exponentially?

\footnotetext{
${ }^{*}$ Corresponding author. E-mail: xuerong@stams.strath.ac.uk
} 
To explain our problem more clearly, let us begin with an example (the notations used will be explained in Section 2). Consider a 2-dimensional regime switching differential equation (also known as a differential equation with Markovian switching or a bybrid differential equation)

$$
\frac{d y(t)}{d t}=z_{r(t)}+Z_{r(t)} y(t), \quad t \geq 0 .
$$

Here $r(t)$ is a right-continuous Markov chain on the state space $\{1,2\}$ with generator

$$
\Gamma=\left(\begin{array}{rr}
-1 & 1 \\
3 & -3
\end{array}\right)
$$

while

$$
z_{1}=\left(\begin{array}{r}
-1 \\
1
\end{array}\right), \quad z_{2}=\left(\begin{array}{r}
1 \\
-1
\end{array}\right), \quad Z_{1}=\left(\begin{array}{rr}
-2 & 1 \\
-1 & -1
\end{array}\right), \quad Z_{2}=\left(\begin{array}{rr}
1 & 1 \\
-1 & 1
\end{array}\right) .
$$

System (1.1) can be regarded as that it operates in two regimes and it obeys

$$
\frac{d y(t)}{d t}=z_{1}+Z_{1} y(t) \quad \text { and } \quad \frac{d y(t)}{d t}=z_{2}+Z_{2} y(t)
$$

in regime 1 and 2 respectively, and the system will switch from one regime to the other according to the probability law of the Markov chain. In Example 2.2 below, we will show that for any initial value $y(0) \in \mathbb{R}^{2}$, the solution of equation (1.1) will obey

$$
\limsup _{t \rightarrow \infty} \frac{\log (|y(t)|)}{\log t} \leq 0.9666 \quad \text { a.s. }
$$

This implies that there is a random variable $T=T(\omega, y(0))>0$ such that

$$
|y(t)| \leq t, \quad \forall t \geq T
$$

with probability one. In other words, the solution of equation (1.1) will, in long term, not grow faster than the time evolves. Let us now perturb equation (1.1) into a hybrid stochastic differential equation (SDE)

$$
d x(t)=\left(z_{r(t)}+Z_{r(t)} x(t)\right) d t+\xi_{r(t)} d B_{1}(t)+\sigma_{r(t)}\left(\begin{array}{r}
x_{2}(t) \\
-x_{1}(t)
\end{array}\right) d B_{2}(t), \quad t \geq 0
$$

where $\sigma_{1}=0$ and $\sigma_{2}>0, \xi_{1}=0, \xi_{2}=\left(\xi_{21}, \xi_{22}\right)^{T}$, and $B_{1}$ and $B_{2}$ are two independent Brownian motions. By setting $\sigma_{1}=0$ and $\xi_{1}=0$, we here indicate the case where in regime 1 there is no white noise while in regime 2 the white noise can be added on. In Example 3.3 below, we show that if $\xi_{2}=(6,0)^{T}$ and $\sigma_{2}^{2}=37$, then the solution of the SDE (1.3) obeys

$$
\liminf _{t \rightarrow \infty} \frac{1}{t} \log (|x(t)|) \geq 0.736 \quad \text { a.s. }
$$

that is, the solution will grow exponentially with probability one. The significant differences between equation (1.1) and its corresponding SDE (1.3) show the important fact that noise expresses exponential growth. 
The problem addressed in this paper is in the area where the study is to find significant differences between a given ordinary differential equation and its corresponding perturbed SDE. The pioneering work in this area was due to Hasminskii [12, p.229], who stabilised an unstable system by using two white noise sources, and his work opened a new chapter in the study of stochastic stabilisation. There is an extensive literature concerned with the stabilisation by noise and we here mention $[1,2,3,4,5,7,9,10,14,17,21,24,25]$. It is now well known that noise can be used to stabilise a given unstable system or to make a system even more stable when it is already stable. A few years ago, Mao et al. [19] showed another important fact that the environmental noise can suppress explosions (in a finite time) in population dynamics and this paper made an important impact on the study of stochastic population systems (see e.g. [8, 13]). Recently, Deng et al. [11] reveal one more important feature that noise can suppress or expresses exponential growth.

However, most of the papers mentioned above consider only the perturbation by white noise but not colour noise yet. In this paper, we will develop the theory presented in [11] to cope with much more general systems where they are subject to both white noise and colour noise. More precisely, we will consider a given system under regime switching (colour noise) whose solution grows at most polynomially, and suppose that the system is subject to environmental noise (white noise) in some regimes. Can the regime switching and the environmental noise work together to make the system change significantly? In this paper we will give a very positive answer. We will first investigate the polynomial growth of hybrid differential equations in Section 2 while, in contrast, discuss the exponential growth of hybrid SDEs in Section 3. Making use of these results we will show that noise can expresses exponential growth under regime switching in Section 4. An important class of linear systems will be discussed in Section 5 while we conclude our paper in Section 6.

\section{Polynomial Growth of Switching Differential Equa- tions}

Throughout the paper, unless otherwise specified, we will employ the following notation. Let $\left(\Omega, \mathcal{F},\{\mathcal{F}(t)\}_{t \geq 0}, \mathbb{P}\right)$ be a complete probability space with a filtration $\{\mathcal{F}(t)\}_{t \geq 0}$ satisfying the usual conditions (i.e., it is increasing and right continuous while $\mathcal{F}(0)$ contains all $\mathbb{P}$-null sets). Let $B(t)=\left(B_{1}(t), \cdots, B_{m}(t)\right)^{T}, t \geq 0$, be an $m$-dimensional Brownian motion defined on the probability space, where $T$ denotes the transpose of a vector or matrix. If $x, y$ are real numbers, then $x \vee y$ denotes the maximum of $x$ and $y$, and $x \wedge y$ denotes the minimum of $x$ and $y$. Let $|x|$ be the Euclidean norm of a vector $x \in \mathbb{R}^{n}$ and $\langle x, y\rangle$ be the inner product of vectors $x, y \in \mathbb{R}^{n}$. Vectors $x \in \mathbb{R}^{n}$ are thought as column ones so to get row vectors we use $x^{T}$. The space of $n \times m$ matrices with real entries is denoted by $\mathbb{R}^{n \times m}$. If $A=\left(a_{i j}\right)$ is an $n \times m$ matrix, we denote its Frobenius or trace norm by

$$
|A|=\sqrt{\sum_{i=1}^{n} \sum_{j=1}^{m} a_{i j}^{2}}
$$


while its operator norm by $\|A\|=\sup \left\{|A x|: x \in \mathbb{R}^{m},|x|=1\right\}$. If $A \in \mathbb{R}^{n \times n}$ is symmetric, its largest and smallest eigenvalue are denoted by $\lambda_{\max }(A)$ and $\lambda_{\min }(A)$, respectively.

Let $r(t), t \geq 0$, be a right-continuous Markov chain on the probability space taking values in a finite state space $\mathbb{S}=\{1,2, \cdots, N\}$ with generator $\Gamma=\left(\gamma_{i j}\right)_{N \times N}$ given by

$$
\mathbb{P}\{r(t+\Delta)=j \mid r(t)=i\}=\left\{\begin{aligned}
\gamma_{i j} \Delta+o(\Delta) & \text { if } i \neq j \\
1+\gamma_{i j} \Delta+o(\Delta) & \text { if } i=j
\end{aligned}\right.
$$

where $\Delta>0$. Here $\gamma_{i j} \geq 0$ is transition rate from $i$ to $j$ if $i \neq j$ while

$$
\gamma_{i i}=-\sum_{j \neq i} \gamma_{i j}
$$

We assume that the Markov chain $r(\cdot)$ is independent of the Brownian motion $B(\cdot)$. It is well known that almost every sample path of $r(t)$ is a right continuous step function. As a standing hypothesis we assume in this paper that the Markov chain is irreducible. This is equivalent to the condition that for any $i, j \in \mathbb{S}$, one can find finite numbers $i_{1}, i_{2}, \cdots, i_{k} \in$ $\mathbb{S}$ such that $\gamma_{i, i_{1}} \gamma_{i_{1}, i_{2}} \cdots \gamma_{i_{k}, j}>0$. Note that $\Gamma$ always has an eigenvalue 0 . The algebraic interpretation of irreducibility is $\operatorname{rank}(\Gamma)=N-1$. Under this condition, the Markov chain has a unique stationary (probability) distribution $\pi=\left(\pi_{1}, \pi_{2}, \cdots, \pi_{N}\right) \in \mathbb{R}^{1 \times N}$ which can be determined by solving the following linear equation

$$
\pi \Gamma=0
$$

subject to

$$
\sum_{j=1}^{N} \pi_{j}=1 \quad \text { and } \quad \pi_{j}>0 \quad \forall j \in \mathbb{S} .
$$

Consider an $n$-dimensional ordinary differential equation with Markovian switching

$$
\frac{d y(t)}{d t}=f(y(t), r(t), t), \quad t \geq 0
$$

As a standing hypothesis, we assume that $f$ is smooth enough so that given any initial values $y(0)=x_{0} \in \mathbb{R}^{n}$ and $r(0)=r_{0} \in \mathbb{S}$, equation (2.1) has a unique solution on $t \geq 0$.

As mentioned in the introduciton section, our key aim of this paper is to reveal that noise expresses exponential growth. For this purpose, let us establish a result that gives conditions under which the solution of equation (2.1) will grow at most polynomially with probability one. It should be pointed out that the research in this direction has its own right. However, our main purpose here is to compare this result with later ones in order to show clearly that it is noise that expresses exponential growth.

Theorem 2.1 Assume that there are constants $h$ and $h_{i}(i \in \mathbb{S})$ such that

$$
2\langle y, f(y, i, t)\rangle \leq h+h_{i}|y|^{2}
$$

for all $(y, i, t) \in \mathbb{R}^{n} \times \mathbb{S} \times \mathbb{R}_{+}$. Assume moreover that there is a constant $\theta \in(0,1)$ such that

$$
-\operatorname{diag}\left(\theta h_{1}, \ldots, \theta h_{N}\right)-\Gamma
$$


is a nonsingular M-matrix. Then the solution of equation (2.1) obeys

$$
\limsup _{t \rightarrow \infty} \frac{\log (|y(t)|)}{\log t} \leq \frac{1}{2 \theta} \quad \text { a.s. }
$$

Proof. By the theory of M-matrices (see e.g. [20, Theorem 2.10 on page 68]), there are positive numbers $q_{1}, \ldots, q_{n}$ such that

$$
\bar{q}_{i}:=-\theta q_{i} h_{i}-\sum_{j=1}^{N} \gamma_{i j} q_{j}>0, \quad i \in \mathbb{S} .
$$

Define

$$
V(y, i)=q_{i}\left(1+|y|^{2}\right)^{\theta}, \quad(y, i) \in \mathbb{R}^{n} \times \mathbb{S} .
$$

By the generalized Itô formula (see e.g. [20, Lemma 1.9 on page 49]),

$$
\mathbb{E} V(y(t), r(t))=V\left(x_{0}, r_{0}\right)+\mathbb{E} \int_{0}^{t} L V(y(s), r(s), s) d s,
$$

where $L V: \mathbb{R}^{n} \times \mathbb{S} \times \mathbb{R}_{+} \rightarrow \mathbb{R}$ is defined by

$$
L V(y, i, t)=q_{i} \theta\left(1+|y|^{2}\right)^{\theta-1}\langle y, f(y, i, t)\rangle+\sum_{j=1}^{N} \gamma_{i j} q_{j}\left(1+|y|^{2}\right)^{\theta} .
$$

By condition (2.2), we compute

$$
\begin{aligned}
L V(y, i, t) \leq & \left(1+|y|^{2}\right)^{\theta-2}\left(q_{i} \theta\left(1+|y|^{2}\right)\left(h+h_{i}|y|^{2}\right)+\sum_{j=1}^{N} \gamma_{i j} q_{j}\left(1+|y|^{2}\right)^{2}\right) \\
= & \left(1+|y|^{2}\right)^{\theta-2} \\
& \times\left(q_{i} \theta\left[h+\left(h+h_{i}\right)|y|^{2}\right]+\sum_{j=1}^{N} \gamma_{i j} q_{j}\left(1+2|y|^{2}\right)-\bar{q}_{i}|y|^{4}\right) .
\end{aligned}
$$

Choose $\varepsilon>0$ sufficiently small for

$$
\varepsilon<\min _{i \in \mathbb{S}} \frac{\bar{q}_{i}}{q_{i}}
$$

Then, by the generalised Itô formula again,

$$
\mathbb{E}\left[e^{\varepsilon t} V(y(t), r(t))\right]=V\left(x_{0}, r_{0}\right)+\mathbb{E} \int_{0}^{t} e^{\varepsilon s}[\varepsilon V(y(s), r(s))+L V(y(s), r(s), s)] d s .
$$

But, by (2.6) and (2.7), we estimate that, for $(y, i, t) \in \mathbb{R}^{n} \times \mathbb{S} \times \mathbb{R}_{+}$,

$$
\begin{aligned}
\varepsilon V(y, i)+L V(y, i, t) \leq & \left(1+|y|^{2}\right)^{\theta-2}\left(q_{i} \theta\left[h+\left(h+h_{i}\right)|y|^{2}\right]\right. \\
& \left.\quad+\left[\varepsilon q_{i}+\sum_{j=1}^{N} \gamma_{i j} q_{j}\right]\left(1+2|y|^{2}\right)-\left(\bar{q}_{i}-\varepsilon q_{i}\right)|y|^{4}\right) \\
\leq & C_{1},
\end{aligned}
$$


where $C_{1}$ is a positive constant. We therefore derive from (2.8) that

$$
\hat{q} \mathbb{E}\left[e^{\varepsilon t}\left(1+|y(t)|^{2}\right)^{\theta}\right] \leq V\left(x_{0}, r_{0}\right)+\int_{0}^{t} C_{1} e^{\varepsilon s} d s \leq V\left(x_{0}, r_{0}\right)+\frac{C_{1}}{\varepsilon} e^{\varepsilon t},
$$

where $\hat{q}=\min _{i \in \mathbb{S}} q_{i}$. This implies that

$$
\mathbb{E}\left[\left(1+|y(t)|^{2}\right)^{\theta}\right] \leq \frac{1}{\hat{q}}\left(V\left(x_{0}, r_{0}\right)+\frac{C_{1}}{\varepsilon}\right):=C_{2} \quad \forall t \geq 0 .
$$

Now, choose $\delta>0$ so small for

$$
4(\delta \check{h})^{\theta} \leq 1,
$$

where $\check{h}=\max \left\{|h|,\left|h_{1}\right|, \cdots,\left|h_{N}\right|\right\}$. Let $k=1,2, \cdots$. For $t \in[k \delta,(k+1) \delta]$, we clearly have

$$
1+|y(t)|^{2}=1+|y(k \delta)|^{2}+\int_{k \delta}^{t} 2\langle y(s), f(y(s), r(s), s)\rangle d s
$$

By condition (2.2),

$$
1+|y(t)|^{2} \leq 1+|y(k \delta)|^{2}+\int_{k \delta}^{t} \check{h}\left(1+|y(s)|^{2}\right) d s,
$$

Noting that for any $a, b \geq 0$

$$
(a+b)^{\theta} \leq[2(a \vee b)]^{\theta} \leq 2\left[a^{\theta} \vee b^{\theta}\right] \leq 2\left[a^{\theta}+b^{\theta}\right],
$$

and using 2.9, we then have

$$
\begin{aligned}
\mathbb{E}\left(\sup _{k \delta \leq t \leq(k+1) \delta}\left(1+|y(t)|^{2}\right)^{\theta}\right) & \leq 2 C_{2}+2 \mathbb{E}\left(\left[\int_{k \delta}^{(k+1) \delta} \check{h}\left(1+|y(s)|^{2}\right) d s\right]^{\theta}\right) \\
& \leq 2 C_{2}+2(\delta \hat{h})^{\theta} \mathbb{E}\left(\sup _{k \delta \leq s \leq(k+1) \delta}\left(1+|y(s)|^{2}\right)^{\theta}\right) .
\end{aligned}
$$

Recalling (2.10), we get

$$
\mathbb{E}\left(\sup _{k \delta \leq t \leq(k+1) \delta}\left(1+|y(t)|^{2}\right)^{\theta}\right) \leq 4 C_{2}
$$

whence

$$
\mathbb{E}\left(\sup _{k \delta \leq t \leq(k+1) \delta}|y(t)|^{2 \theta}\right) \leq 4 C_{2}, \quad \forall k \geq 1 .
$$

Let $\bar{\varepsilon}>0$ be arbitrary. Then, by the well-known Chebyshev inequality, we have

$$
\mathbb{P}\left\{\sup _{k \delta \leq t \leq(k+1) \delta}|y(t)|^{2 \theta}>(k \delta)^{1+\bar{\varepsilon}}\right\} \leq \frac{4 C_{2}}{(k \delta)^{1+\bar{\varepsilon}}}, \quad k=1,2, \cdots
$$

Applying the well-known Borel-Cantelli lemma (see e.g. [18]), we obtain that for almost all $\omega \in \Omega$,

$$
\sup _{k \delta \leq t \leq(k+1) \delta}|y(t)|^{2 \theta} \leq(k \delta)^{1+\bar{\varepsilon}}
$$


holds for all but finitely many $k$. Hence, there exists a $k_{0}(\omega)$, for almost all $\omega \in \Omega$, for which (2.13) holds whenever $k \geq k_{0}$. Consequently, for almost all $\omega \in \Omega$, if $k \geq k_{0}$ and $k \delta \leq t \leq(k+1) \delta$,

$$
\frac{\log \left(|y(t)|^{2 \theta}\right)}{\log t} \leq \frac{(1+\bar{\varepsilon}) \log (k \delta)}{\log (k \delta)}=1+\bar{\varepsilon}
$$

Therefore

$$
\limsup _{t \rightarrow \infty} \frac{\log (|y(t)|)}{\log t} \leq \frac{1+\bar{\varepsilon}}{2 \theta} \quad \text { a.s. }
$$

Letting $\bar{\varepsilon} \rightarrow 0$ we obtain that

$$
\limsup _{t \rightarrow \infty} \frac{\log (|y(t)|)}{\log t} \leq \frac{1}{2 \theta} \quad \text { a.s. }
$$

which is the desired assertion (2.4). The proof is therefore complete.

Example 2.2 Let us consider an $n$-dimensional hybrid differential equation

$$
\frac{d y(t)}{d t}=z_{r(t)}+Z_{r(t)} y(t), \quad t \geq 0 .
$$

Here $r(t)$ is a right-continuous Markov chain on the state space $\mathbb{S}=\{1,2\}$ with generator

$$
\Gamma=\left(\begin{array}{rr}
-\gamma_{12} & \gamma_{12} \\
\gamma_{21} & -\gamma_{21}
\end{array}\right), \quad\left(\gamma_{12}>0, \gamma_{21}>0\right)
$$

while $z:\{1,2\} \rightarrow \mathbb{R}^{n}$ and $Z:\{1,2\} \rightarrow \mathbb{R}^{n \times n}$. Assume that

$$
\lambda_{\max }\left(Z_{1}+Z_{1}^{T}\right)<0, \quad \lambda_{\max }\left(Z_{2}+Z_{2}^{T}\right)>0, \quad \frac{\gamma_{21}}{\lambda_{\max }\left(Z_{2}+Z_{2}^{T}\right)}>\frac{\gamma_{12}}{\left|\lambda_{\max }\left(Z_{1}+Z_{1}^{T}\right)\right|} .
$$

Define

$$
f(x, i, t)=z_{i}+Z_{i} x, \quad(x, i, t) \in \mathbb{R}^{n} \times \mathbb{S} \times \mathbb{R}_{+} .
$$

Then equation (2.14) becomes (2.1). Compute, for any $\theta \in(0,1)$,

$$
\begin{aligned}
2\langle x, f(x, i, t)\rangle & =2\left\langle x, z_{i}\right\rangle+\left\langle x,\left(Z_{i}+Z_{i}^{T}\right) x\right\rangle \\
& \leq 2\left|z_{i}\right||x|+\lambda_{\max }\left(Z_{i}+Z_{i}^{T}\right)|x|^{2} \\
& \leq \frac{\left|z_{i}\right|^{2}}{\theta}+\left(\lambda_{\max }\left(Z_{i}+Z_{i}^{T}\right)+\theta\right)|x|^{2} .
\end{aligned}
$$

In other words, condition (2.2) is satisfied with

$$
h_{i}=\lambda_{\max }\left(Z_{i}+Z_{i}^{T}\right)+\theta, \quad i \in \mathbb{S} .
$$

The matrix defined by (2.3) becomes

$$
\left(\begin{array}{cc}
\theta\left(\left|\lambda_{\max }\left(Z_{1}+Z_{1}^{T}\right)\right|-\theta\right)+\lambda_{12} & \lambda_{12} \\
\lambda_{21} & -\theta\left(\lambda_{\max }\left(Z_{2}+Z_{2}^{T}\right)+\theta\right)+\lambda_{21}
\end{array}\right) .
$$

Clearly we can choose $\theta$ sufficiently small for

$$
\theta\left(\left|\lambda_{\max }\left(Z_{1}+Z_{1}^{T}\right)\right|-\theta\right)+\lambda_{12}>0 \quad \text { and } \quad-\theta\left(\lambda_{\max }\left(Z_{2}+Z_{2}^{T}\right)+\theta\right)+\lambda_{21}>0 .
$$


Hence, matrix (2.16) is a non-singular M-matrix if and only if

$$
\left[\theta\left(\left|\lambda_{\max }\left(Z_{1}+Z_{1}^{T}\right)\right|-\theta\right)+\lambda_{12}\right]\left[-\theta\left(\lambda_{\max }\left(Z_{2}+Z_{2}^{T}\right)+\theta\right)+\lambda_{21}\right]-\lambda_{12} \lambda_{21}>0,
$$

namely

$$
\begin{aligned}
\psi(\theta):= & -\theta\left(\left|\lambda_{\max }\left(Z_{1}+Z_{1}^{T}\right)\right|-\theta\right)\left(\lambda_{\max }\left(Z_{2}+Z_{2}^{T}\right)+\theta\right) \\
& +\lambda_{21}\left(\left|\lambda_{\max }\left(Z_{1}+Z_{1}^{T}\right)\right|-\theta\right)-\lambda_{12}\left(\lambda_{\max }\left(Z_{2}+Z_{2}^{T}\right)+\theta\right)>0 .
\end{aligned}
$$

Clearly, $\psi(\theta)$ is a continuous function on $\theta \in[0,1)$ and, by condition (2.15),

$$
\psi(0)=\lambda_{21}\left|\lambda_{\max }\left(Z_{1}+Z_{1}^{T}\right)\right|-\lambda_{12} \lambda_{\max }\left(Z_{2}+Z_{2}^{T}\right)>0 .
$$

Hence, we can further, if necessary, choose $\theta$ sufficiently small for $\psi(\theta)>0$. In other words, condition (2.15) guarantees that there is a $\theta \in(0,1)$ for matrix $(2.16)$ to be a non-singular M-matrix. By Theorem 2.1, we can therefore conclude that for any initial value $y(0) \in \mathbb{R}^{n}$, the solution of equation (2.14) will obey

$$
\limsup _{t \rightarrow \infty} \frac{\log (|y(t)|)}{\log t} \leq \frac{1}{2 \theta} \quad \text { a.s. }
$$

In other words, under condition (2.15), the solution of equation (2.14) will grow at most polynomially with probability one.

Let us return to equation (1.1). Recall that $\gamma_{12}=1, \gamma_{21}=3$ and compute

$$
\lambda_{\max }\left(Z_{1}+Z_{1}^{T}\right)=-2 \quad \lambda_{\max }\left(Z_{2}+Z_{2}^{T}\right)=2 .
$$

Hence condition (2.15) is satisfied by equation (1.1). We therefore know that the solution of equation (1.1) will grow at most polynomially with probability one. To be more preciese, we observe that matrix $(2.16)$ becomes

$$
\left(\begin{array}{cc}
\theta(2-\theta)+1 & 1 \\
3 & -\theta(2+\theta)+3
\end{array}\right) .
$$

Clearly, $\theta(2-\theta)+1>0$ and $-\theta(2+\theta)+3>0$ when $\theta \in(0,1)$. So, for the matrix above to be a non-singular M-matrix, all we need is a $\theta \in(0,1)$ for

$$
[\theta(2-\theta)+1][-\theta(2+\theta)+3]-3>0
$$

namely

$$
\theta^{3}-8 \theta+4>0
$$

It is easy to see that this holds whenever $0<\theta<0.5173$, whence $(2.18)$ holds. We can then conclude that the solution of equqtion (1.1) obeys

$$
\limsup _{t \rightarrow \infty} \frac{\log (|y(t)|)}{\log t} \leq \frac{1}{2 \times 0.5173}=0.9666 \quad \text { a.s. }
$$




\section{Exponential Growth of SDEs}

Let us now stochastically perturb the differential equation (2.1) into a stochastic differential equation (SDE)

$$
d x(t)=f(x(t), r(t), t) d t+g(x(t), r(t), t) d B(t)
$$

on $t \geq 0$ with the initial data $x(0)=x_{0} \in \mathbb{R}^{n}$ and $r(0)=r_{0} \in \mathbb{S}$, where

$$
f: \mathbb{R}^{n} \times \mathbb{S} \times \mathbb{R}_{+} \rightarrow \mathbb{R}^{n} \quad \text { and } \quad g: \mathbb{R}^{n} \times \mathbb{S} \times \mathbb{R}_{+} \rightarrow \mathbb{R}^{n \times m}
$$

Our aim here is to show how the stochastic perturbation will force the solution to grow exponentially, in constrast to the fact that the solution of the corresponding differential equation (2.1) will only grow at most polynomially.

Again, as a standing hypothesis in this paper, we assume that the coefficients $f$ and $g$ are sufficiently smooth so that the SDE (3.1) has a unique global solution $x(t)$ on $t \in \mathbb{R}_{+}$. For example, a known condition for this is that both coefficients $f$ and $g$ are locally Lipschitz continuous and obey the linear growth condition (see e.g. [20]).

In particular, under the linear growth condition: there is a positive constant $H$ such that

$$
|f(x, i, t)|^{2} \vee|g(x, i, t)|^{2} \leq H\left(1+|x|^{2}\right), \quad \forall(x, i, t) \in \mathbb{R}^{n} \times \mathbb{S} \times \mathbb{R}_{+},
$$

then the solution obeys

$$
\limsup _{t \rightarrow \infty} \frac{1}{t} \log (|x(t)|) \leq \sqrt{H}+\frac{H}{2} \quad \text { a.s. }
$$

(see e.g. [20, Theorem 3.17 on page 93]). That is, the solution will grow at most exponentially with probability one. However, there is so far no result on the lower bound for

$$
\liminf _{t \rightarrow \infty} \frac{1}{t} \log (|x(t)|) .
$$

In this section, we will establish some sufficient conditions under which we have

$$
\liminf _{t \rightarrow \infty} \frac{1}{t} \log (|x(t)|)>0 \quad \text { a.s. }
$$

that is, the solution will grow exponentially with probability one.

Theorem 3.1 Assume that for each $i \in \mathbb{S}$, there are constants $c_{1 i}-c_{6 i}$ such that

$$
-c_{1 i}-c_{2 i}|x|^{2} \leq 2\langle x, f(x, i, t)\rangle, \quad\left|x^{T} g(x, i, t)\right|^{2} \leq c_{3 i}|x|^{2}+c_{4 i}|x|^{4}
$$

and

$$
|g(x, i, t)|^{2} \geq c_{5 i}+c_{6 i}|x|^{2}
$$

for all $(x, t) \in \mathbb{R}^{n} \times \mathbb{R}_{+}$. Set

$$
\alpha_{i}=c_{5 i}-c_{1 i}, \quad \beta_{i}=c_{5 i}+c_{6 i}-c_{1 i}-c_{2 i}-2 c_{3 i}, \quad \gamma_{i}=c_{6 i}-c_{2 i}-2 c_{4 i},
$$


and define

$$
\delta_{i}=\left\{\begin{array}{cc}
\alpha_{i} \wedge \gamma_{i} & \text { if } \beta_{i} \geq 2\left(\alpha_{i} \wedge \gamma_{i}\right) \\
\min \left\{\alpha_{i}, \gamma_{i}, \frac{\alpha_{i} \gamma_{i}-0.25 \beta_{i}^{2}}{\alpha_{i}+\gamma_{i}-\beta_{i}}\right\} & \text { otherwise }
\end{array}\right.
$$

If

$$
\bar{\delta}:=\sum_{i \in \mathbb{S}} \pi_{i} \delta_{i}>0
$$

then the solution of equation (3.1) obeys

$$
\liminf _{t \rightarrow \infty} \frac{1}{t} \log (|x(t)|) \geq \frac{\bar{\delta}}{2} \quad \text { a.s. }
$$

To prove this theorem, let us present a lemma.

Lemma 3.2 Let $\alpha, \beta$ and $\gamma$ be three real numbers and define $\delta=\alpha \wedge \gamma$ if $\beta \geq 2(\alpha \wedge \gamma)$ or otherwise

$$
\delta=\min \left\{\alpha, \gamma, \frac{\alpha \gamma-0.25 \beta^{2}}{\alpha+\gamma-\beta}\right\}
$$

Then

$$
\alpha+\beta u+\gamma u^{2} \geq \delta(1+u)^{2}, \quad \forall u \geq 0
$$

Proof. If $\beta \geq 2(\alpha \wedge \gamma)$, then, for $u \geq 0$,

$$
\alpha+\beta u+\gamma u^{2} \geq \delta+2 \delta u+\delta u^{2}=\delta(1+u)^{2},
$$

which is (3.8). Let us now consider the case when $\beta<2(\alpha \wedge \gamma)$. Write, for $u \geq 0$,

$$
\begin{aligned}
\alpha+\beta u+\gamma u^{2}-\delta(1+u)^{2} & =\alpha-\delta+(\beta-2 \delta) u+(\gamma-\delta) u^{2} \\
& =(1, u)\left(\begin{array}{cc}
\alpha-\delta & 0.5 \beta-\delta \\
0.5 \beta-\delta & \gamma-\delta
\end{array}\right)\left(\begin{array}{l}
1 \\
u
\end{array}\right) .
\end{aligned}
$$

By the definition of $\delta$, we know $\alpha-\delta \geq 0$ and $\gamma-\delta \geq 0$. It is therefore clear that (3.8) will hold if

$$
(\alpha-\delta)(\gamma-\delta) \geq(0.5 \beta-\delta)^{2}
$$

namely

$$
(\alpha+\gamma-\beta) \delta \leq \alpha \gamma-0.25 \beta^{2}
$$

But, given that $\beta<2(\alpha \wedge \gamma)$, we must have $\beta<\alpha+\gamma$. Hence, the inequality above is equivalent to

$$
\delta \leq \frac{\alpha \gamma-0.25 \beta^{2}}{\alpha+\gamma-\beta}
$$

which is guaranteed by the definition of $\delta$. In other words, we have showed that the assertion (3.8) holds as well when $\beta<2(\alpha \wedge \gamma)$.

We can now prove Theorem 3.1.

Proof. By the Itô formula we compute

$$
d\left[\log \left(1+|x(t)|^{2}\right)\right]=G(x(t), r(t), t) d t+\frac{2 x^{T}(t) g(x(t), r(t), t)}{1+|x(t)|^{2}} d B(t),
$$


where $G: \mathbb{R}^{n} \times \mathbb{S} \times \mathbb{R}_{+} \rightarrow \mathbb{R}$ is defined by

$$
G(x, i, t)=\frac{2\langle x, f(x, i, t)\rangle+|g(x, i, t)|^{2}}{1+|x|^{2}}-\frac{2\left|x^{T} g(x, i, t)\right|^{2}}{\left(1+|x|^{2}\right)^{2}} .
$$

By conditions (3.2) and (3.3),

$$
\begin{aligned}
G(x, i, t) & \geq \frac{\left(c_{5 i}-c_{1 i}\right)+\left(c_{6 i}-c_{2 i}\right)|x(t)|^{2}}{1+|x|^{2}}-\frac{2 c_{3 i}|x(t)|^{2}+2 c_{4 i}|x(t)|^{4}}{\left(1+|x|^{2}\right)^{2}} \\
& =\frac{F\left(|x|^{2}, i\right)}{\left(1+|x(t)|^{2}\right)^{2}},
\end{aligned}
$$

where $F: \mathbb{R}_{+} \times \mathbb{S} \rightarrow \mathbb{R}$ is defined by

$$
F(u, i)=\alpha_{i}+\beta_{i} u+\gamma_{i} u^{2},
$$

in which the parameters $\alpha_{i}, \beta_{i}$ and $\gamma_{i}$ have been defined by (3.4). By Lemma 3.2,

$$
F(u, i) \geq \delta_{i}(1+u)^{2}, \quad \forall u \geq 0,
$$

where $\delta_{i}$ is defined by (3.5). We therefore obtain from (3.9) that

$$
d\left[\log \left(1+|x(t)|^{2}\right)\right] \geq \delta_{r(t)} d t+\frac{2 x^{T}(t) g(x(t), r(t), t)}{1+|x(t)|^{2}} d B(t),
$$

whence

$$
\log \left(1+|x(t)|^{2}\right) \geq \log \left(1+\left|x_{0}\right|^{2}\right)+\int_{0}^{t} \delta_{r(s)} d s+M(t)
$$

where

$$
M(t)=\int_{0}^{t} \frac{2 x^{T}(s) g(x(s), r(s), s)}{1+|x(s)|^{2}} d B(s),
$$

which is a continuous martingale with initial value $M(0)=0$. By condition (3.2), we compute its quadratic variation

$$
\langle M(t)\rangle=\int_{0}^{t} \frac{4\left|x^{T}(s) g(x(s), r(s), s)\right|}{1+|x(s)|^{2}} d t \leq \int_{0}^{t} \frac{4\left(\check{c}_{3}|x(s)|^{2}+\check{c}_{4}|x(s)|^{4}\right)}{1+|x(s)|^{2}} d t \leq 4\left(\check{c}_{3}+\check{c}_{4}\right) t,
$$

where $\check{c}_{3}=\max _{i \in \mathbb{S}} c_{3 i}$ and $\check{c}_{4}=\max _{i \in \mathbb{S}} c_{4 i}$. Hence, by the strong law of large numbers of martingales (see e.g. [18, Theorem 3.4 on page 12]),

$$
\lim _{t \rightarrow \infty} \frac{M(t)}{t}=0 \quad \text { a.s. }
$$

Moreover, by the well-known ergodic theory of the Markov chain,

$$
\lim _{t \rightarrow \infty} \frac{1}{t} \int_{0}^{t} \delta_{r(s)} d s=\sum_{i \in \mathbb{S}} \pi_{i} \delta_{i}=\bar{\delta} \quad \text { a.s. }
$$

Dividing both sides of (3.12) and then letting $t \rightarrow \infty$ we then obtain

$$
\liminf _{t \rightarrow \infty} \frac{1}{t} \log \left(1+|x(t)|^{2}\right) \geq \bar{\delta} \quad \text { a.s. }
$$


Hence, for any $\varepsilon \in(0, \bar{\delta})$, there exists a random number $T=T(\omega)>0$ such that, for almost all $\omega \in \Omega$,

$$
\frac{1}{t} \log \left(1+|x(t, \omega)|^{2}\right) \geq \bar{\delta}-\varepsilon, \quad t \geq T(\omega) .
$$

That is,

$$
|x(t, \omega)|^{2} \geq e^{(\bar{\delta}-\varepsilon) t}-1, \quad t \geq T(\omega) .
$$

This implies

$$
\liminf _{t \rightarrow \infty} \frac{1}{t} \log (|x(t, \omega)|) \geq \frac{\bar{\delta}-\varepsilon}{2} .
$$

As $\varepsilon>0$ is arbitrary, we must have

$$
\liminf _{t \rightarrow \infty} \frac{1}{t} \log (|x(t, \omega)|) \geq \frac{\bar{\delta}}{2}
$$

for almost all $\omega \in \Omega$ as required.

Let us make some comments on the conditions of Theorem 3.1. We observe that condition (3.2) can be satisfied by a large class of functions. For example, if both $f$ and $g$ obey the linear growth condition

$$
|f(x, i, t)|^{2} \vee|g(x, i, t)|^{2} \leq H\left(1+|x|^{2}\right),
$$

then

$$
-2\langle x, f(x, i, t)\rangle \leq 2|x||f(x, i, t)| \leq 2 \sqrt{H}\left(|x|+|x|^{2}\right) \leq \sqrt{H}\left(1+|x|^{2}\right)
$$

and

$$
\left|x^{T} g(x, i, t)\right|^{2} \leq|x|^{2}|g(x, i, t)|^{2} \leq H\left(|x|^{2}+|x|^{4}\right),
$$

that is $f$ and $g$ obey (3.2). However, instead of using the linear growth condition, the forms described in (3.2) enable us to compute the parameters $c_{1 i}-c_{4 i}$ more precisely in many situations as illustrated in the examples discussed in the next sections. In the following sections we will show clearly that condition (3.3) associated with noise is very critical in order to have an exponential growth.

Example 3.3 Let us consider the SDE (1.3). Given (1.2), it is easy to obtain the stationary distribution of the Markov chain on the state space $\mathbb{S}=\{1,2\}$ is $\pi=\left(\pi_{1}, \pi_{2}\right)=$ $(3 / 4,1 / 4)$. For $(x, i, t) \in \mathbb{R}^{2} \times \mathbb{S} \times \mathbb{R}_{+}$, define

$$
f(x, i, t)=z_{i}+Z_{i} x, \quad g(x, 1, t)=0, \quad g(x, 2, t)=\left(\begin{array}{rr}
\xi_{21} & \sigma_{2} x_{2} \\
\xi_{22} & -\sigma_{2} x_{1}
\end{array}\right) .
$$

Then equation (1.3) becomes (3.1). It is easy to show

$$
\begin{gathered}
-2-5|x|^{2} \leq 2\langle x, f(x, 1, t)\rangle, \quad-2-\left(-|x|^{2}\right) \leq 2\langle x, f(x, 2, t)\rangle, \\
\left|x^{T} g(x, 1, t)\right|^{2}=0, \quad\left|x^{T} g(x, 2, t)\right|^{2} \leq\left|\xi_{2}\right|^{2}|x|^{2}, \\
|g(x, 1, t)|^{2}=0, \quad|g(x, 2, t)|^{2} \geq\left|\xi_{2}\right|^{2}+\sigma_{2}^{2}|x|^{2} .
\end{gathered}
$$

That is, (3.2) and (3.3) hold with

$$
c_{11}=2, c_{21}=5, c_{31}=c_{41}=c_{51}=c_{61}=0,
$$




$$
c_{12}=2, c_{22}=-1, c_{32}=\left|\xi_{2}\right|^{2}, c_{42}=0, c_{52}=\left|\xi_{2}\right|^{2}, c_{62}=\sigma_{2}^{2} .
$$

Hence, the parameters defined by (3.4) are

$$
\alpha_{1}=-2, \beta_{1}=-7, \gamma_{1}=-5, \alpha_{2}=\left|\xi_{2}\right|^{2}-2, \beta_{2}=\sigma_{2}^{2}-\left|\xi_{2}\right|^{2}-1, \gamma_{2}=\sigma_{2}^{2}+1 .
$$

By (3.5), we get $\delta_{1}=-5$. Now choose $\xi_{2}$ for $\left|\xi_{2}\right|^{2}>2$ and choose $\sigma_{2}=\left|\xi_{2}\right|^{2}+1$. Then, by $(3.5)$, we get

$$
\delta_{2}=\frac{\left|\xi_{2}\right|^{4}-4}{2\left|\xi_{2}\right|^{2}}
$$

Choose $\xi_{2}>15+\sqrt{229}$ for

$$
\bar{\delta}=-\frac{15}{4}+\frac{\left|\xi_{2}\right|^{4}-4}{8\left|\xi_{2}\right|^{2}}>0 .
$$

Then, by Theorem 3.1, the solution of the SDE (1.3) obeys

$$
\liminf _{t \rightarrow \infty} \frac{1}{t} \log (|x(t)|) \geq \frac{\bar{\delta}}{2} \quad \text { a.s. }
$$

For instance, let $\xi_{2}=(6,0)^{T}$ and $\sigma_{2}^{2}=37$. Then

$$
\liminf _{t \rightarrow \infty} \frac{1}{t} \log (|x(t)|) \geq 0.736 \quad \text { a.s. }
$$

\section{Noise Expresses Exponential Growth}

In the above sections, we have discussed the polynomial growth of the differential equation (2.1) and the exponential growth of the corresponding SDE (3.1). The question is: Given equation (2.1) can we design the diffusion coefficient $g$ so that the solution of the SDE (3.1) will grow exponentially with probability one? Example 3.3 discussed in the above section shows the possibility. In this section we will develop the idea there into a general theory. We will assume, implicitly, that the conditions of Theorem 2.1 hold. In this way, we show clearly that it is noise that expresses exponential growth.

In order to make use of Theorem 3.1, we naturally impose the following condition

$$
-c_{1 i}-c_{2 i}|x|^{2} \leq\langle x, f(x, i, t)\rangle, \quad(x, i, t) \in \mathbb{R}^{n} \times \mathbb{S} \times \mathbb{R}_{+},
$$

where $c_{1 i}$ and $c_{2 i}$ are non-negative constants. This condition gives a lower bound for $\langle x, f(x, i, t)\rangle$ while $(2.2)$ gives an upper bound.

We will design $g$ to be independent of $t$ so we will write $g(x, i, t)$ as $g(x, i)$ in this section. Moreover, we shall see that the noise term $g(x(t), r(t)) d B(t)$ can be designed to be a linear form of $x(t)$.

\subsection{Even dimension of the state space}

First, let the dimension of the state space $n$ be an even number and choose the dimension of the Brownian motion $m$ to be 2 . Design $g: \mathbb{R}^{n} \times \mathbb{S} \rightarrow \mathbb{R}^{n \times 2}$ by

$$
g(x, i)=\left(\xi_{i}, A_{i} x\right)
$$


where $\xi_{i}=\left(\xi_{i 1}, \cdots, \xi_{\text {in }}\right)^{T} \in \mathbb{R}^{n}$ and

$$
A_{i}=\left(\begin{array}{ccccc}
0 & \sigma_{i} & & & \\
-\sigma_{i} & 0 & & & \\
& & \ddots & & \\
& & & 0 & \sigma_{i} \\
& & & -\sigma_{i} & 0
\end{array}\right)
$$

with $\sigma_{i} \in \mathbb{R}$. So the stochastically perturbed system (3.1) becomes

$$
d x(t)=f(x(t), r(t), t) d t+\xi_{r(t)} d B_{1}(t)+\sigma_{r(t)}\left(\begin{array}{c}
x_{2}(t) \\
-x_{1}(t) \\
\vdots \\
x_{n}(t) \\
-x_{n-1}(t)
\end{array}\right) d B_{2}(t)
$$

For $(x, i) \in \mathbb{R}^{n} \times \mathbb{S}$, compute

$$
\left|x^{T} g(x, i)\right|^{2}=\left(x^{T} \xi_{i}\right)^{2}+\left(x^{T} A_{i} x\right)^{2}=\left(x^{T} \xi_{i}\right)^{2} \leq\left|\xi_{i}\right|^{2}|x|^{2}
$$

and

$$
|g(x, i)|^{2}=\left|\xi_{i}\right|^{2}+\left|A_{i} x\right|^{2}=\left|\xi_{i}\right|^{2}+\sigma_{i}^{2}|x|^{2} .
$$

That is, conditions (3.2) and (3.3) are satisfied with

$$
c_{3 i}=\left|\xi_{i}\right|^{2}, \quad c_{4 i}=0, \quad c_{5 i}=\left|\xi_{i}\right|^{2}, \quad c_{6 i}=\sigma_{i}^{2} .
$$

Consequently, the parameters defined by (3.4) becomes

$$
\alpha_{i}=\left|\xi_{i}\right|^{2}-c_{1 i}, \quad \beta_{i}=\sigma_{i}^{2}-c_{1 i}-c_{2 i}-\left|\xi_{i}\right|^{2}, \quad \gamma_{i}=\sigma_{i}^{2}-c_{2 i} .
$$

There are various ways to choose $\xi_{i}$ and $\sigma_{i}$ for the solution of equation (4.2) to grow exponentially with probability one. Let us discuss a number of cases.

Case 4.1.1. For each $i \in \mathbb{S}$, choose

$$
\left|\xi_{i}\right|^{2}>c_{1 i} \text { and } \sigma_{i}^{2}=c_{1 i}+c_{2 i}+\left|\xi_{i}\right|^{2}
$$

Then $\beta_{i}=0$ and $\gamma_{i}=\left|\xi_{i}\right|^{2}+c_{1 i} \geq \alpha_{i}$, and

$$
\frac{\alpha_{i} \gamma_{i}-0.25 \beta_{i}^{2}}{\alpha_{i}+\gamma_{i}-\beta_{i}}=\frac{\left|\xi_{i}\right|^{4}-c_{1 i}^{2}}{2\left|\xi_{i}\right|^{2}} \leq \frac{\alpha_{i}\left(\left|\xi_{i}\right|^{2}+c_{1 i}\right)}{2\left|\xi_{i}\right|^{2}}<\alpha_{i} .
$$

whence, the $\delta_{i}$ defined by (3.5) becomes

$$
\delta_{i}=\frac{\left|\xi_{i}\right|^{4}-c_{1 i}^{2}}{2\left|\xi_{i}\right|^{2}}>0 .
$$

By Theorem 3.1, the solution of equation (4.2) obeys

$$
\liminf _{t \rightarrow \infty} \frac{1}{t} \log (|x(t)|) \geq \sum_{i \in \mathbb{S}} \frac{\pi_{i}\left(\left|\xi_{i}\right|^{2}-c_{1 i}^{2}\right)}{4|\xi|^{2}}>0 . \quad \text { a.s. }
$$


Case 4.1.2. For each $i \in \mathbb{S}$, choose

$$
\left|\xi_{i}\right|^{2}>c_{1 i} \quad \text { and } \quad \sigma_{i}^{2}=3\left|\xi_{i}\right|^{2}+c_{2 i}-c_{1 i}
$$

Then $\gamma_{i}=3\left|\xi_{i}\right|^{2}-c_{1 i}=2\left|\xi_{i}\right|^{2}+\alpha_{i}>\alpha_{i}, \beta_{i}=2 \alpha_{i}$, so the $\delta_{i}$ defined by (3.5) becomes

$$
\delta_{i}=\alpha_{i}=\left|\xi_{i}\right|^{2}-c_{1 i}>0 .
$$

By Theorem 3.1, the solution of equation (4.2) obeys

$$
\liminf _{t \rightarrow \infty} \frac{1}{t} \log (|x(t)|) \geq \sum_{i \in \mathbb{S}} \frac{\pi_{i}}{2}\left(\left|\xi_{i}\right|^{2}-c_{1 i}\right)>0 \quad \text { a.s. }
$$

Case 4.1.3. Divide $\mathbb{S}$ into two parts, namely $\mathbb{S}=\mathbb{S}_{1} \cup \mathbb{S}_{2}$. Choose $\xi_{i}$ and $\sigma_{i}$ by (4.4) for each $i \in \mathbb{S}_{1}$ while by (4.6) for each $i \in \mathbb{S}_{2}$. It is then easy to see that the solution of equation (4.2) obeys

$$
\liminf _{t \rightarrow \infty} \frac{1}{t} \log (|x(t)|) \geq \sum_{i \in \mathbb{S}_{1}} \frac{\pi_{i}\left(\left|\xi_{i}\right|^{2}-c_{1 i}^{2}\right)}{4\left|\xi_{i}\right|^{2}}+\sum_{i \in \mathbb{S}_{2}} \frac{\pi_{i}}{2}\left(\left|\xi_{i}\right|^{2}-c_{1 i}\right)>0 \quad \text { a.s. }
$$

Case 4.1.4. Again divide $\mathbb{S}$ into two parts, namely $\mathbb{S}=\mathbb{S}_{1} \cup \mathbb{S}_{2}$, but assume that the system is not obserable or cannot be perturbed stochastically in any regime $i \in \mathbb{S}_{1}$. In other words, we have to set $\xi_{i}=0$ and $\sigma_{i}=0$ for every $i \in \mathbb{S}_{1}$ but we can choose $\xi_{i}$ and $\sigma_{i}$ for $i \in \mathbb{S}_{2}$. Accordingly, for each $i \in \mathbb{S}_{1}$,

$$
c_{3 i}=c_{4 i}=c_{5 i}=c_{6 i}=0, \quad \alpha_{i}=-c_{1 i}, \quad \beta_{i}=-c_{1 i}-c_{2 i}, \quad \gamma_{i}=-c_{2 i} .
$$

Clearly, $\beta_{i}=-c_{1 i}-c_{2 i} \geq 2\left(\left(-c_{1 i}\right) \wedge\left(-c_{2 i}\right)\right)=2\left(\alpha_{i} \wedge \gamma_{i}\right)$ so, by $(3.5)$,

$$
\delta_{i}=\left(-c_{1 i}\right) \wedge\left(-c_{2 i}\right) .
$$

On the other hand, for each $i \in \mathbb{S}_{2}$, choose $\xi_{i}$ and $\sigma_{i}$ by (4.6), and, in addition, choose $\xi_{i}$ sufficiently large for

$$
\sum_{i \in \mathbb{S}_{1}} \pi_{i}\left[\left(-c_{1 i}\right) \wedge\left(-c_{2 i}\right)\right]+\sum_{i \in \mathbb{S}_{2}} \frac{\pi_{i}}{2}\left(\left|\xi_{i}\right|^{2}-c_{1 i}\right)>0
$$

Then, by Theorem 3.1, the solution of equation (4.2) obeys

$$
\liminf _{t \rightarrow \infty} \frac{1}{t} \log (|x(t)|) \geq \sum_{i \in \mathbb{S}_{1}} \pi_{i}\left[\left(-c_{1 i}\right) \wedge\left(-c_{2 i}\right)\right]+\sum_{i \in \mathbb{S}_{2}} \frac{\pi_{i}}{2}\left(\left|\xi_{i}\right|^{2}-c_{1 i}\right)>0 \quad \text { a.s. }
$$

\subsection{Odd dimension of the state space}

We next let the dimension of the state space $n$ be an odd number but $n \geq 3$. Choose the dimension of the Brownian motion $m$ to be 3 and design $g: \mathbb{R}^{n} \times \mathbb{S} \rightarrow \mathbb{R}^{n \times 3}$ by

$$
g(x, i)=\left(\xi_{i}, F_{i} x, G_{i} x\right)
$$


where $\xi \in \mathbb{R}^{n}$ while

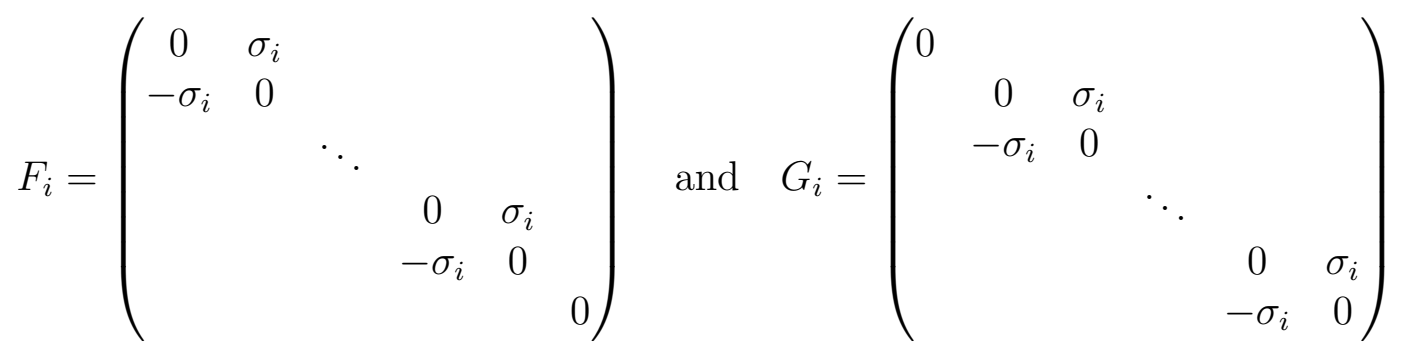

with $\sigma_{i} \geq 0$. So the stochastically perturbed system (3.1) becomes

$$
d x(t)=f(x(t), r(t), t) d t+\xi_{r(t)} d B_{1}+\sigma_{r(t)}\left(\begin{array}{c}
x_{2}(t) \\
-x_{1}(t) \\
\vdots \\
x_{n-1}(t) \\
-x_{n-2}(t) \\
0
\end{array}\right) d B_{2}(t)+\sigma_{r(t)}\left(\begin{array}{c}
0 \\
x_{2}(t) \\
-x_{3}(t) \\
\vdots \\
x_{n}(t) \\
-x_{n-1}(t)
\end{array}\right) d B_{3}(t) .
$$

For $(x, i) \in \mathbb{R}^{n} \times \mathbb{S}$, compute

$$
\left.\mid x^{T} g(x, i)\right)\left.\right|^{2}=\left(x^{T} \xi_{i}\right)^{2}+\left(x^{T} F_{i} x\right)^{2}+\left(x^{T} G_{i} x\right)^{2}=\left(x^{T} \xi_{i}\right)^{2} \leq\left|\xi_{i}\right|^{2}|x|^{2}
$$

and

$$
\mid g(x, i))\left.\right|^{2}=\left|\xi_{i}\right|^{2}+\left|F_{i} x\right|^{2}+\left|G_{i} x\right|^{2} \geq\left|\xi_{i}\right|^{2}+\sigma_{i}^{2}|x|^{2} .
$$

Hence, conditions (3.2) and (3.3) are satisfied with the same parameters specified by (4.3). Hence, the conclusions obtained in Cases 4.1.1-4 apply to the perturbed SDE (4.10).

Summarizing the above arguments, we obtain the following result.

Theorem 4.1 Any nonlinear hybrid differential equation $\dot{y}(t)=f(y(t), r(t), t)$ can be stochastically perturbed by Brownian motions into the hybrid SDE (3.1) whose solutions will grow exponentially with probability one provided (4.1) is satisfied and the dimension of the state space is greater than 1. The result still holds even in the case where stochastic perturbation is only allowed in some regimes. Moreover, the noise term $g(x(t), r(t), t) d B(t)$ in (3.1) can be designed to be a linear form of $x(t)$.

\subsection{Scalar case}

The theorem above requires the dimension of the state space be greater than 1 . Naturally, we may wonder what happens in the scalar case? More precisely, the question is: Given a scalar swithcing differential equation $\dot{y}(t)=f(y(t), r(t), t)$ whose solutions grow at most polynomially, can we stochastically perturb it into a hybrid SDE whose solutions will grow exponentially with probability one?

To answer this question, let us consider a linear hybrid differential equation

$$
\dot{y}(t)=p(r(t))-q(r(t)) y(t), \quad t \geq 0 .
$$


Assume that $p(i)$ and $q(i)(i \in \mathbb{S})$ are all positive numbers. It can be showed by Theorem 2.1 that the solution of equation (4.11) will grow at most polynomially with probability one. Let us now stochastically perturb this equation into a

$$
d x(t)=(p(r(t))-q(r(t)) x(t)) d t+\sum_{k=1}^{m}\left(u_{k}(r(t))+v_{k}(r(t)) x(t)\right) d B_{k}(t),
$$

where $u_{k}(i)$ and $v_{k}(i)$ are all real numbers. We will show in elsewhere, due to the page limit here, that for any given $u_{k}(i)$ and $v_{k}(i)$, the solution of this SDE will still grow at most polynomially with probability one. In other words, the linear stochastic perturbation $\sum_{k=1}^{m}\left(u_{k}(r(t))+v_{k}(r(t)) x(t)\right) d B_{k}(t)$ may not force the solution of a scalar system $\dot{y}(t)=$ $f(y(t), r(t), t)$ to grow exponentially.

\section{$5 \quad$ Linear Systems}

Let us consider an $n$-dimensional linear hybrid differential equation

$$
\frac{d y(t)}{d t}=u(r(t))+U(r(t)) y(t), \quad t \geq 0,
$$

where $u: \mathbb{S} \rightarrow \mathbb{R}^{n}$ and $U: \mathbb{S} \rightarrow \mathbb{R}^{n \times n}$, and we will write $u(i)=u_{i}$ and $U(i)=U_{i}$. This is a special case of eqution (2.1) where

$$
f(y, i, t)=u_{i}+U_{i} y, \quad(y, i, t) \in \mathbb{R}^{n} \times \mathbb{S} \times \mathbb{R}_{+} .
$$

Compute

$$
\left.|\langle y, f(y, i, t) \mid\rangle \leq| y^{T} u_{i}|+| y^{T} U_{i} y|\leq| u_{i}|| y\left|+\left\|U_{i}\right\|\right| y\right|^{2} \leq 0.5\left|u_{i}\right|+\left(0.5\left|u_{i}\right|+\| U_{i}||\right)|y|^{2},
$$

whence

$$
-0.5\left|u_{i}\right|-\left(0.5\left|u_{i}\right|+\left\|U_{i}\right\|\right)|y|^{2} \leq\langle y, f(y, i, t)\rangle \leq 0.5\left|u_{i}\right|+\left(0.5\left|u_{i}\right|+\left\|U_{i}\right\|\right)|y|^{2} .
$$

In ohter words, condition (4.1) holds with $c_{1 i}=0.5\left|u_{i}\right|$ and $c_{2 i}=0.5\left|u_{i}\right|+\left\|U_{i}\right\|$.

Now, divide $\mathbb{S}$ into two parts, namely $\mathbb{S}=\mathbb{S}_{1} \cup \mathbb{S}_{2}$. Assume that the system is not observable or cannot be perturbed stochastically in any regime $i \in \mathbb{S}_{1}$ but observable or can be perturbed stochastically in every regime $i \in \mathbb{S}_{2}$.

\section{Case 5.1 Even dimension}

When the dimension of the state space is even, we can stochastically perturb system (5.1) into

$$
d x(t)=[u(r(t))+U(r(t)) x(t)] d t+\xi_{r(t)} d B_{1}(t)+\sigma_{r(t)}\left(\begin{array}{c}
x_{2}(t) \\
-x_{1}(t) \\
\vdots \\
x_{n}(t) \\
-x_{n-1}(t)
\end{array}\right) d B_{2}(t),
$$


where $\xi_{i}=0$ and $\sigma_{i}=0$ for $i \in \mathbb{S}_{1}$, while $\xi_{i} \in \mathbb{R}^{n}$ and $\sigma_{i} \in \mathbb{R}$ for $i \in \mathbb{S}_{2}$. Based on the result in Case 4.1.4, if we choose $\xi_{i}$ and $\sigma_{i}\left(i \in \mathbb{S}_{2}\right)$ such that

$$
\left|\xi_{i}\right|^{2}>0.5\left|u_{i}\right|, \quad \sigma_{i}^{2}=3\left|\xi_{i}\right|^{2}+\left\|U_{i}\right\|
$$

and

$$
\sum_{i \in \mathbb{S}_{2}} \frac{\pi_{i}}{2}\left(\left|\xi_{i}\right|^{2}-0.5\left|u_{i}\right|\right)>\sum_{i \in \mathbb{S}_{1}} \pi_{i}\left(0.5\left|u_{i}\right|+\left\|U_{i}\right\|\right),
$$

then the solutions of the stochastically perturbed system (5.2) will grow exponentially with probability one.

\section{Case 5.2 Odd dimension}

When the dimension of the state space is odd and greater than 2 , we can stochastically perturb system (5.1) into

$$
\begin{aligned}
d x(t)= & {[u(r(t))+U(r(t)) x(t)] d t+\xi_{r(t)} d B_{1} } \\
& +\sigma_{r(t)}\left(\begin{array}{c}
x_{2}(t) \\
-x_{1}(t) \\
\vdots \\
x_{n-1}(t) \\
-x_{n-2}(t) \\
0
\end{array}\right) d B_{2}(t)+\sigma_{r(t)}\left(\begin{array}{c}
0 \\
x_{2}(t) \\
-x_{3}(t) \\
\vdots \\
x_{n}(t) \\
-x_{n-1}(t)
\end{array}\right) d B_{3}(t),
\end{aligned}
$$

where $\xi_{i}$ and $\sigma_{i}$ are chosen in the same way as in Case 5.1. Then, based on the result in Section 4.2, the solutions of the stochastically perturbed system (5.3) will grow exponentially with probability one.

\section{Conclusions}

In this paper, we consider a system described by a differential equation under regime switching, whose solution may grow at most polynomially. We suppose that the system is subject to environmental noise in some regimes, or we can stochastically perturb the system in some regimes. We then show that the regime switching and the environmental noise will make the original system whose solution grows at most polynomially become a new system whose solutions will grow exponentially. In other words, we reveal that the regime switching and the environmental noise will express the exponential growth.

However, everything has two sides. To close our paper we would like to point out that the regime switching and the environmental noise may also suppress exponential growth. But, due to the page limit here, we will report these results elsewhere.

\section{Acknowledgements}

This research was carried out when X. Mao visited University of Science and Technology Beijing and Harbin Institute of Technology. The authors would like to thank both Universities for their hospitality and partial financial support. The authors would also like 
to thank the financial support from the National Natural Science Foundation of China (grant number 10571036).

\section{References}

[1] Appleby, J.A.D., Stabilisation of functional differential equations by noise, Systems Control Lett., to appear.

[2] Appleby, J.A.D., Destabilisation of functional differential equations by noise, Systems Control Lett., to appear.

[3] Appleby, J.A.D. and Flynn, A., Stabilisation of Volterra equations by noise, J. Appl. Math. Stoch. Anal., to appear.

[4] Appleby, J.A.D. and Mao, X., Stochastic stabilisation of functional differential equations, System Control Lett. 54(11) (2005), 1069-1081.

[5] Appleby, J.A.D., Mao, X. and Rodkina, A., On stochastic stabilisation of difference equations, Discrete and Continuous Dynamical Systems-Series A 15(3) (2006), 843857.

[6] Arnold, L., Random Dynamical Systems, Springer, 1998.

[7] Arnold, L., Crauel, H. and Wihstutz, V., Stabilisation of linear systems by noise, SIAM J. Control Optim. 21 (1983), 451-461.

[8] Bahar, A. and Mao, X., Stochastic delay Lotka-Volterra model, J. Math. Anal. Appl. 292 (2004), 364-380.

[9] Bellman, R., Bentsman, J. and Meerkov, S., Stability of fast periodic systems, IEEE Tans. Automa. Control 30 (1985), 289-291.

[10] Caraballo, T., Garrido-Atienza, M. and Real, J. Stochastic stabilisation of differential systems with general decay rate. Systems Control Lett. 48 (2003), 397-406.

[11] Deng, F., Luo, Q., Mao, X. and Pang, S., Noise suppresses or expresses exponential growth, Systems and Control Letters 57 (2008), 262-270.

[12] Hasminskii, R.Z., Stochastic Stability of Differential Equations, Sijthoff and Noordhoff, 1981.

[13] Jiang, D.Q. and Shi, N.Z., A note on nonautonomous logistic equation with random perturbation, J. Math. Anal. Appl. 303 (2005), 977-986.

[14] Kushner, H. J., On the stability of processes defined by stochastic differencedifferential equations, J. Differential Equations 4 (1968), 424-443.

[15] Mao, X., Stability of Stochastic Differential Equations with Respect to Semimartingales, Longman Scientific and Technical, 1991. 
[16] Mao, X., Exponential Stability of Stochastic Differential Equations, Marcel Dekker, 1994.

[17] Mao, X., Stochastic stabilisation and destabilisation, Systems and Control Letters 23 (1994), 279-290.

[18] Mao, X., Stochastic Differential Equations and Their Applications, 2nd Edition, Chichester: Horwood Pub., 2007.

[19] Mao, X., Marion, G. and Renshaw, E., Environmental noise suppresses explosion in population dynamics, Stochastic Processes and Their Applications 97 (2002), 95-110.

[20] Mao, X. and Yuan, C., Stochastic Differential Equations with Markovian Switching, Imperial College Press, 2006.

[21] Meerkov, S., Condition of vibrational stabilizability for a class of non-linear systems, IEEE Trans. Automat. Control 27 (1982), 485-487.

[22] Minkowski, H., Diophantische Approximationen, Teubner, Leipzig, 1907.

[23] Pinsky, M.A. and Wihstutz, V., Lyapunov exponents of nilponent Itô systems, Stochastics 25 (1988), 43-57.

[24] Scheutzow, M., Stabilisation and destabilisation by noise in the plane, Stoch. Anal. Appl. 11(1) (1993), 97-113.

[25] Zhabko, A.P. and Kharitonov, V.L., Problem of vibrational stabilisation of linear systems, Automat. Telemekh. 2 (1980), 31-34. 